Annals of Pure and Applied Mathematics

Vol. 16, No. 1, 2018, 181-191

ISSN: 2279-087X (P), 2279-0888(online)

Published on 12 January 2018

WwW.researchmathsci.org

DOI: http://dx.doi.org/10.22457/apam.v16n1a20

Annals of

Pure and Applied

Mathematics

\title{
Monotone Iterative Technique for Caputo Fractional Differential Equations with Deviating Arguments
}

\author{
D. B. Dhaigude ${ }^{1}$ and B. H. Rizqan ${ }^{2}$
}

Department of Mathematics, Dr. Babasaheb Ambedkar Marathwada University

Aurangabad - 431004 (M.S.) India

Email: 1ㅁyyanraja@gmail.com, ${ }^{2}$ bakeralhaaiti@yahoo.com

${ }^{2}$ Corresponding author

Received 4 November 2017; accepted 6 December 2017

Abstract. In this paper, we obtain the existence and uniqueness of solution for fractional differential equation involving the Caputo fractional derivative with deviating argument. The uniqueness of solution is obtained by using a Banach fixed point theorem also the existence of extremal solutions are obtained by a monotone iterative technique and the method of lower and upper solutions. Finally, some examples illustrate the results.

Keywords: Fractional differential equation with deviating argument, Caputo fractional derivative, existence and uniqueness, monotone iterative technique, extremal solutions.

AMS Mathematics Subject Classification (2010): 34A08, 34A12, 26A33, 34L15

\section{Introduction}

In this paper, we study the following problem for nonlinear initial value problem (IVP) involving Caputo fractional differential equation with deviating argument:

$$
\left\{\begin{aligned}
{ }^{C} D_{0+}^{\alpha} x(t) & =f(t, x(t), x(\theta(t))), t \in J=[0, T], \\
x(0) & =x_{0}, x^{\prime}(0)=0,
\end{aligned}\right.
$$

where $f(t, x(t), x(\theta(t))) \in C\left([0, T] \times \mathbb{R}^{2}, \mathbb{R}\right), \theta \in C(J, J), \theta(t) \leq t, t \in J,{ }^{C} D_{0+}^{\alpha}$ is called the Caputo fractional derivative of order $\alpha \quad(1<\alpha \leq 2)$.

Since $f(t, x(t), x(\theta(t)))$ is continuous, IVP (1) is equivalent to the following Volterra fractional integral equation

$$
x(t)=x_{0}+\frac{1}{\Gamma(\alpha)} \int_{0}^{t}(t-s)^{\alpha-1} f(s, x(s), x(\theta(s))) d s .
$$

Where $\Gamma$ denotes the gamma function, recently, the theory of fractional differential equation has a lot of importance recently because of its applications (see $[8,20])$. In the recent investigations, many researchers studied the existence and uniqueness of solution of nonlinear fractional differential equations (see [2, 3, 4, 5, 12, 15, 17] and references therein). Also, there was an important development in fractional differential equation see $[7,9,14,19,22]$ and the papers of $[6,12,16,18]$. Very recently, was discussed some 


\section{B. Dhaigude and B. H. Rizqan}

basic theory for nonlinear IVP of fractional differential equation involving the RiemannLiouville differential operator of order $\alpha(0<\alpha<1)$ (see $[10,11]$ and the references therein). However, discussion on initial value problems of fractional differential equation with deviating argument is rare. We know that it is important build a comparison result relative to lower and upper solutions of Caputo which we using in the monotone iterative technique for initial value problem of order $\alpha(1<\alpha \leq 2)$.

The paper is organized as follows: In Section 2, we present some definitions and fundamental facts of fractional calculus theory. In Section 3, we will prove the uniqueness of solution for nonlinear IVP (1) by using Banach fixed point theorem. In Section 4 , by the utility of the monotone iterative technique and the method of lower and upper solutions, we prove that nonlinear IVP (1) has extremal solutions. Lastly, we illustrate our results with suitable examples.

\section{Preliminaries}

Let $C(J, \mathbb{R})=\{x: x(t)$ is continuous on $C(J, \mathbb{R})\}$ with the norm $\|x\|_{C}=\max _{t \in J}|x(t)|$.

Obviously, $C(J, \mathbb{R})$ is Banach space. For the convenience of the readers, we first present some useful definitions and fundamental facts of fractional calculus theory, which can be found in $[7,19]$.

Definition 2.1. Caputo's derivative for a function $f(t) \in C^{n}(J, \mathbb{R})$ can be written as

$$
{ }_{0}^{C} D_{t}^{\alpha} f(t)=\left(I_{0+}^{n-\alpha} D^{n} f\right)(t)=\frac{1}{\Gamma(n-\alpha)} \int_{0}^{t} \frac{f^{(n)}(s) d s}{(t-s)^{\alpha+1-n}},
$$

where $D=\frac{d}{d t}$ and $n=[\alpha]+1,[\alpha]$ denotes the integer part of real number $\alpha>0$.

Definition 2.2. For $\alpha>0$, the integral

$$
I_{0+}^{\alpha} f(t)=\frac{1}{\Gamma(\alpha)} \int_{0}^{t}(t-s)^{\alpha-1} f(s) d s,
$$

is called the Riemann-Liouville fractional integral operator of order $\alpha$.

Lemma 2.1. [7] Let $x(t) \in C^{n}[0,1]$ and $\alpha \in(n-1, n], n \in \mathbb{N}$. Then for $t \in[0,1]$,

$$
I_{0+}^{\alpha{ }^{C}} D_{0+}^{\alpha} x(t)=x(t)-\sum_{k=0}^{n-1} \frac{t^{k}}{k !} x^{k}(0) .
$$

\section{Uniqueness of solution}

In this section, we discuss uniqueness of solution for nonlinear IVP (1) for Caputo fractional differential equation with deviating argument under the following condition:

$\left(H_{1}\right)$ There exist nonnegative constants $L_{1}, L_{2}$ such that

$$
\left|f\left(t, v_{1}, v_{2}\right)-f\left(t, u_{1}, u_{2}\right)\right| \leq L_{1}\left|v_{1}-u_{1}\right|+L_{2}\left|v_{2}-u_{2}\right|, \forall t \in J, v_{i}, u_{i} \in \mathbb{R}, i=1,2 .
$$


Monotone Iterative Technique for Caputo Fractional Differential Equations ...

Lemma 3.1. Let $f \in C\left(J \times \mathbb{R}^{2}, \mathbb{R}\right), x \in C(J, \mathbb{R})$ is the solution of the nonlinear IVP (1), if and only if $x(t)$ is a solution of integral equation

$$
x(t)=x_{0}+\frac{1}{\Gamma(\alpha)} \int_{0}^{t}(t-s)^{\alpha-1} f(s, x(s), x(\theta(s))) d s .
$$

Proof: Assume that $x(t)$ satisfies IVP (1). From the first equation of IVP (1) and Lemma 2.1, we have

$$
x(t)=x_{0}+\frac{1}{\Gamma(\alpha)} \int_{0}^{t}(t-s)^{\alpha-1} f(s, x(s), x(\theta(s))) d s .
$$

Conversely, assume that $x(t)$ satisfies (3). Applying the operator ${ }_{0}^{C} D_{t}^{\alpha}$ to both sides of (3), we have

$$
{ }_{0}^{C} D_{t}^{\alpha} x(t)=f(t, x(t), x(\theta(t))) .
$$

In addition, we have $x(0)=x_{0}, x^{\prime}(0)=0$. The proof is complete.

Theorem 3.1. Assume that $\left(H_{1}\right)$ hold, $f \in C\left(J \times \mathbb{R}^{2}, \mathbb{R}\right)$. Then the nonlinear IVP (1) has a unique solution.

Proof: Define the operator $A: C(J, \mathbb{R}) \rightarrow C(J, \mathbb{R})$ by

$$
(A x)(t)=x_{0}+\frac{1}{\Gamma(\alpha)} \int_{0}^{t}(t-s)^{\alpha-1} f(s, x(s), x(\theta(s))) d s .
$$

It is easy to check that the operator $(A x)(t) \in C(J, \mathbb{R})$. Next we show that $A$ is a contraction operator $C(J, \mathbb{R})$. For convenience, let

$$
\frac{\left(L_{1}+L_{2}\right) T^{\alpha}}{\Gamma(1+\alpha)}<1
$$

For any $x, y \in C(J, \mathbb{R})$, we have

$$
\begin{aligned}
\|A x-A y\|_{C} & =\max _{t \in J}|(A x)(t)-(A y)(t)| \\
& \leq \max _{t \in J} \frac{1}{\Gamma(\alpha)} \int_{0}^{t}(t-s)^{\alpha-1}|f(s, x(s), x(\theta(s)))-f(s, y(s), y(\theta(s)))| d s \\
& \leq \frac{1}{\Gamma(\alpha)} \max _{t \in J} \int_{0}^{t}(t-s)^{\alpha-1}\left[L_{1}|x(s)-y(s)|+L_{2}|x(\theta(s))-y(\theta(s))|\right] d s \\
& \leq \frac{1}{\Gamma(\alpha)} \max _{t \in J} \int_{0}^{t}(t-s)^{\alpha-1}\left[L_{1}\|x-y\|_{C}+L_{2}\|x-y\|_{C}\right] d s \\
& \leq \frac{\left(L_{1}+L_{2}\right)}{\Gamma(\alpha)}\|x-y\|_{C} \max _{t \in J} \int_{0}^{t}(t-s)^{\alpha-1} d s \\
& \leq \frac{\left(L_{1}+L_{2}\right)}{\Gamma(\alpha)}\|x-y\|_{C} \max _{t \in J} t^{\alpha} \int_{0}^{1}(1-\eta)^{\alpha-1} d \eta
\end{aligned}
$$




$$
\begin{aligned}
& \leq\left\{\left(\frac{L_{1}+L_{2}}{\Gamma(\alpha)}\right)\left(\frac{\Gamma(\alpha) T^{\alpha}}{\Gamma(1+\alpha)}\right)\right\}\|x-y\|_{C} \\
& \leq \frac{\left(L_{1}+L_{2}\right) T^{\alpha}}{\Gamma(1+\alpha)}\|x-y\|_{C} .
\end{aligned}
$$

According to (4) and using Banach fixed point theorem, the nonlinear IVP (1) has a unique solution. The proof is complete.

Lemma 3.2. Let $M, N \geq 0$ are constants, $h \in C(J, \mathbb{R})$. The linear initial value problem:

$$
\left\{\begin{array}{c}
{ }^{C} D_{0+}^{\alpha} x(t)-M x(t)-N x(\theta(t))=h(t), 1<\alpha \leq 2, t \in[0, T], \\
x(0)=x_{0}, \quad x^{\prime}(0)=0,
\end{array}\right.
$$

has a unique solution

$$
x(t)=x_{0}+\frac{1}{\Gamma(\alpha)} \int_{0}^{t}(t-s)^{\alpha-1}[M x(s)+N x(\theta(s))+h(s)] d s .
$$

Proof: By Theorem 3.1, the linear IVP (5) is equivalent to solving a fixed point problem with operator $A_{h}$ defined by

$$
A_{h} x(t)=x_{0}+\frac{1}{\Gamma(\alpha)} \int_{0}^{t}(t-s)^{\alpha-1}[M(s) x(s)+N(s) x(\theta(s))+h(s)] d s .
$$

For any $h \in C_{1-\alpha}(J)$. Then the operator $A_{h}$ has a unique fixed point.

\section{Remark 1.}

i. Putting $N=0$, in the above linear IVP (5), we have the result obtained by Devi [1], Mcrae [13], Sambandham et al., [21];

ii. Putting $N=0$ and $h(t) \equiv 0$, in the above linear IVP (5), we get the solution of the corresponding homogenous IVP (5) by Yakar [23];

iii. When $\alpha=1$, the problem (5) reduces to the following

$$
\left\{\begin{array}{c}
x^{\prime}(t)=M x(t)+N x(\theta(t))+h(t), t \in J, \\
x(0)=x_{0}, \quad x^{\prime}(0)=0,
\end{array}\right.
$$

has a unique solution which satisfies Eq.(6);

iv. Putting $M=0$ and $N=0$, in the above linear IVP (5), the initial value problem (5) has a unique solution

$$
x(t)=x_{0}+\frac{1}{\Gamma(\alpha)} \int_{0}^{t}(t-s)^{\alpha-1} h(s) d s .
$$

\section{The monotone iterative technique}

In this section, we mainly prove the existence of extremal solutions of the nonlinear IVP (1) by monotone iterative technique combined with the method of lower and upper solutions. We need the following Lemma and definition. 
Monotone Iterative Technique for Caputo Fractional Differential Equations ...

Lemma 4.1. Suppose that $M, N \geq 0$ are constants and the inequality

$$
\frac{(M+N) T^{\alpha}}{\Gamma(1+\alpha)}<1
$$

holds, and $p \in C(J, \mathbb{R})$ satisfies

$$
\begin{aligned}
& \qquad \begin{array}{l}
p(t) \leq p(0)+\frac{1}{\Gamma(\alpha)} \int_{0}^{t}(t-s)^{\alpha-1}[M p(s)+N p(\theta(s))] d s, t \in J, \\
p(0) \leq 0, \\
\text { then } p(t) \leq 0 \text { for all } t \in J .
\end{array}
\end{aligned}
$$

Proof: Suppose that $p(t) \leq 0, \forall t \in J$ is not true. So there exists at least one $t_{*} \in J$ such that $p\left(t_{*}\right)>0$. Without loss of generality, we assume

$$
p\left(t_{*}\right)=\max _{t \in J}\{p(t)\}=\rho_{1}>0 \text {. }
$$

We obtain that

$$
\begin{aligned}
p(t) & \leq p(0)+\frac{1}{\Gamma(\alpha)} \int_{0}^{t}(t-s)^{\alpha-1}[M p(s)+N p(\theta(s))] d s \\
& \leq \frac{1}{\Gamma(\alpha)} \int_{0}^{t}(t-s)^{\alpha-1}[M p(s)+N p(\theta(s))] d s \\
& \leq \frac{M}{\Gamma(\alpha)} \int_{0}^{t}(t-s)^{\alpha-1} p(s) d s+\frac{N}{\Gamma(\alpha)} \int_{0}^{t}(t-s)^{\alpha-1} p(\theta(s)) d s .
\end{aligned}
$$

Let $t=t_{*}$, we have

So

$$
\rho_{1} \leq\left(\frac{(M+N)}{\Gamma(1+\alpha)} T^{\alpha}\right) \rho_{1}
$$

$$
\frac{(M+N)}{\Gamma(1+\alpha)} T^{\alpha} \geq 1
$$

This is a contradiction. Hence $p(t) \leq 0$ for all $t \in J$. The proof is complete.

Definition 4.1. We say that $\alpha_{0} \in C(J, \mathbb{R})$ is called a lower solution of IVP (1) if

$$
\left\{\begin{array}{l}
\alpha_{0}(t) \leq \alpha_{0}(0)+\frac{1}{\Gamma(\alpha)} \int_{0}^{t}(t-s)^{\alpha-1} f\left(s, \alpha_{0}(s), \alpha_{0}(\theta(s))\right) d s, t \in J, \\
\alpha_{0}(0) \leq \alpha_{0}, \alpha_{0}^{\prime}(0) \leq 0
\end{array}\right.
$$

We say that $\beta_{0} \in C(J, \mathbb{R})$ is called a upper solution of IVP (1) if

$$
\left\{\begin{array}{l}
\beta_{0}(t) \geq \beta_{0}(0)+\frac{1}{\Gamma(q)} \int_{0}^{t}(t-s)^{\alpha-1} f\left(s, \beta_{0}(s), \beta_{0}(\theta(s))\right) d s, t \in J, \\
\beta_{0}(0) \geq \beta_{0}, \quad \beta_{0}^{\prime}(0) \geq 0 .
\end{array}\right.
$$




\section{B. Dhaigude and B. H. Rizqan}

In the following discussion, we need the following assumptions:

$\left(H_{2}\right)$ Functions $\alpha_{0}$ and $\beta_{0}$ are ordered lower and upper solutions of nonlinear IVP (1) such that

$$
\alpha_{0}(t) \leq \beta_{0}(t), t \in J
$$

$\left(H_{3}\right)$ There exist constants $M, N \geq 0$ such that

$$
f\left(t, v_{1}, v_{2}\right)-f\left(t, u_{1}, u_{2}\right) \geq M\left(v_{1}-u_{1}\right)+N\left(v_{2}-u_{2}\right),
$$

where $\alpha_{0}(t) \leq u_{1} \leq v_{1} \leq \beta_{0}(t), \alpha_{0}(\theta(t)) \leq u_{2} \leq v_{2} \leq \beta_{0}(\theta(t)), t \in J$.

Let $\left[\alpha_{0}, \beta_{0}\right]=\left\{z \in C(J, \mathbb{R}): \alpha_{0}(t) \leq z(t) \leq \beta_{0}(t), t \in J, \alpha_{0}(0) \leq z(0) \leq \beta_{0}(0)\right\}$.

Theorem 4.1. Let $\left(H_{2}\right)-\left(H_{3}\right)$ and inequality (7) hold. Then there exist two monotone sequences $\left\{\alpha_{n}\right\},\left\{\beta_{n}\right\} \subset\left[\alpha_{0}, \beta_{0}\right]$ both converge uniformly to the extremal solutions of nonlinear IVP (1) in $\left[\alpha_{0}, \beta_{0}\right]$.

Proof: This proof consists of the following three steps.

Step 1: Construct two the sequences $\left\{\alpha_{n}\right\}$ and $\left\{\beta_{n}\right\}$. For any $\eta \in\left[\alpha_{0}, \beta_{0}\right]$ such that $\eta \in C(J, \mathbb{R})$, we consider the following linear initial value problem:

$$
\left\{\begin{array}{c}
{ }^{C} D_{0+}^{\alpha} x(t)-M x(t)-N x(\theta(t))=f(t, \eta(t), \eta(\theta(t)))-M \eta(t)-N \eta(\theta(t)), t \in J, \\
x(0)=x_{0}, \quad x^{\prime}(0)=0 .
\end{array}\right.
$$

In the present context $h(t)=f(t, \eta(t), \eta(\theta(t)))-M \eta(t)-N \eta(\theta(t))$ and is to be replaced in (5) by this new value. By Lemma 3.2, the linear IVP (9) has a unique solution

$$
x(t)=x_{0}+\frac{1}{\Gamma(\alpha)} \int_{0}^{t}(t-s)^{\alpha-1}[M(s) x(s)+N(s) x(\theta(s))+h(s)] d s,
$$

Suppose that $x_{1}(t)$ and $x_{2}(t)$ be two solutions of linear IVP (9). Let $p(t)=x_{1}(t)-x_{2}(t)$. Applying Lemma 4.1 again one can prove that $p(t) \leq 0$, and thus $x_{1}(t) \leq x_{2}(t)$. As the same argument is valid for $x_{2}(t)-x_{1}(t)$, we conclude that $x_{1}(t)=x_{2}(t)$. This proves uniqueness.

Now, we define a mapping $A:\left[\alpha_{0}, \beta_{0}\right] \rightarrow\left[\alpha_{0}, \beta_{0}\right]$ by $x=A \eta$, where $x$ is the unique solution of linear IVP (9) It is easy to check that the operator $A$ is monotone nondecreasing on $\left[\alpha_{0}, \beta_{0}\right]$, let $\eta, \mu \in\left[\alpha_{0}, \beta_{0}\right]$ such that $\eta \leq \mu$. Suppose that $z_{1}=A \eta$ and $z_{2}=A \mu$. Setting $p(t)=z_{1}(t)-z_{2}(t)$, we obtain

$$
\begin{aligned}
p(t) & =p(0)+\frac{1}{\Gamma(\alpha)} \int_{0}^{t}(t-s)^{\alpha-1}\left(\begin{array}{c}
f(s, \eta(s), \eta(\theta(s)))-f(s, \mu(s), \mu(\theta(s))) \\
-M\left(\eta(s)-z_{1}(s)\right)-N\left(\eta(\theta(s))-z_{1}(\theta(s))\right) \\
+M\left(\mu(s)-z_{2}(s)\right)+N\left(\mu(\theta(s))-z_{2}(\theta(s))\right)
\end{array}\right) d s \\
& \leq p(0)+\frac{1}{\Gamma(\alpha)} \int_{0}^{t}(t-s)^{\alpha-1}(M p(s)+N p(\theta(s))) d s .
\end{aligned}
$$


Monotone Iterative Technique for Caputo Fractional Differential Equations ...

Besides,

$$
p(0)=z_{1}(0)-z_{2}(0)=z_{0}-z_{0} \leq 0 .
$$

By Lemma 4.1, we get $p(t) \leq 0$, implies that $A \eta \leq A \mu$ for all $t \in J$. It means that $A$ is monotone nondecreasing on $\left[\alpha_{0}, \beta_{0}\right]$. Obviously, we can easily get that $A$ is a continuous map. It is now easy to define sequences $\left\{\alpha_{n}\right\}$ and $\left\{\beta_{n}\right\}$ such that $\alpha_{n}=A \alpha_{n-1}, \quad \beta_{n}=A \beta_{n-1}, n=1,2, \ldots$

Step 2: The sequences $\left\{\alpha_{n}\right\}$ and $\left\{\beta_{n}\right\}$ converge uniformly to $\alpha^{*}, \beta^{*}$ respectively. In fact $\left\{\alpha_{n}\right\}$ and $\left\{\beta_{n}\right\}$ satisfy the following relation:

$$
\alpha_{0} \leq \alpha_{1} \leq \cdots \leq \alpha_{n} \leq \cdots \alpha^{*} \leq \beta^{*} \cdots \leq \beta_{n} \leq \cdots \leq \beta_{1} \leq \beta_{0} .
$$

Setting $p(t)=\alpha_{0}(t)-\alpha_{1}(t)$ and $\alpha_{0}(t)$ is the lower solution of IVP (1), we obtain

$$
\begin{aligned}
p(t) & \leq p(0)+\frac{1}{\Gamma(\alpha)} \int_{0}^{t}(t-s)^{\alpha-1}\left[\begin{array}{c}
f\left(s, \alpha_{0}(s), \alpha_{0}(\theta(s))\right)-f\left(s, \alpha_{0}(s), \alpha_{0}(\theta(s))\right) \\
+M\left(\alpha_{0}(s)-\alpha_{1}(s)\right)+N\left(\alpha_{0}(\theta(s))-\alpha_{1}(\theta(s))\right)
\end{array}\right] d s \\
& \leq p(0)+\frac{1}{\Gamma(\alpha)} \int_{0}^{t}(t-s)^{\alpha-1}(M p(s)+N p(\theta(s))) d s .
\end{aligned}
$$

Besides,

$$
p(0)=\alpha_{0}(0)-\alpha_{1}(0) \leq \alpha_{0}-\alpha_{0}=0 .
$$

By Lemma 4.1, we get $p(t) \leq 0$, implies that $\alpha_{0}(t) \leq \alpha_{1}(t)$ for all $t \in J$. Similarly, we can show that $\beta_{1} \leq \beta_{0}$ for all $t \in J$. Applying the operator $A$ to both sides of $\alpha_{0} \leq \alpha_{1}$, $\beta_{1} \leq \beta_{0}, \alpha_{1} \leq \beta_{1}$ and $\alpha_{0} \leq \beta_{0}$, we can easily get (11). Obviously, the sequences $\left\{\alpha_{n}\right\}$ and $\left\{\beta_{n}\right\}$ are uniformly bounded and equicontinuous on $J$. Then by using the AscoliArzela criterion, we can conclude that the sequences $\left\{\alpha_{n}\right\}$ and $\left\{\beta_{n}\right\}$ converge uniformly on $J$ with $\lim _{n \rightarrow \infty} \alpha_{n}=\alpha^{*}, \lim _{n \rightarrow \infty} \beta_{n}=\beta^{*}$ uniformly on $J$.

Step 3: Prove that $\alpha^{*}, \beta^{*}$ are extremal solutions of nonlinear IVP (1) and $\alpha^{*}, \beta^{*}$ are solutions of nonlinear IVP (1) on $\left[\alpha_{0}, \beta_{0}\right]$ because of the continuity of operator $A$. Let $z \in\left[\alpha_{0}, \beta_{0}\right]$ be any solution of nonlinear IVP (1). That is,

$$
\left\{\begin{array}{l}
z(t)=z(0)+\frac{1}{\Gamma(\alpha)} \int_{0}^{t}(t-s)^{\alpha-1} f(s, z(s), z(\theta(s))) d s, \\
z(0)=z_{0}, z^{\prime}(0)=0 .
\end{array}\right.
$$

Suppose that there exists a positive integer $n$ such that $\alpha_{n}(t) \leq z(t) \leq \beta_{n}(t)$ on $J$. Let $p(t)=\alpha_{n+1}(t)-z(t)$, we obtain 


$$
\begin{aligned}
& p(t)=p(0)+\frac{1}{\Gamma(\alpha)} \int_{0}^{t}(t-s)^{\alpha-1}\left(\begin{array}{c}
f\left(s, \alpha_{n}(s), \alpha_{n}(\theta(s))\right)-f(s, z(s), z(\theta(s))) \\
-M\left(\alpha_{n}(s)-\alpha_{n+1}(s)\right)-N\left[\alpha_{n}(\theta(s))-\alpha_{n+1}(\theta(s))\right]
\end{array}\right) d s \\
& p(t) \leq p(0)+\frac{1}{\Gamma(\alpha)} \int_{0}^{t}(t-s)^{\alpha-1}(M p(s)+N p(\theta(s))) d s .
\end{aligned}
$$

Besides,

$$
p(0)=\alpha_{n+1}(0)-z(0)=\alpha_{n}-z_{0} \leq 0 .
$$

By Lemma 4.1, we get $p(t) \leq 0$, implies $\alpha_{n+1}(t) \leq z(t)$ for all $t \in J$. Similarly, we obtain that $z(t) \leq \beta_{n+1}(t)$ on $J$.

Since $\alpha_{0}(t) \leq z(t) \leq \beta_{0}(t)$ on $J$, by induction we get that $\alpha_{n}(t) \leq z(t) \leq \beta_{n}(t)$ on $J$ for all $n$. Therefore, $\alpha^{*}(t) \leq z(t) \leq \beta^{*}(t)$ on $[0, T]$ by taking $n \rightarrow \infty$. The proof is complete.

\section{Examples}

Example 5.1. Consider the following problem:

$$
\left\{\begin{array}{c}
{ }^{C} D_{0+}^{\frac{3}{2}} x(t)=\frac{1}{30} x(t)+\frac{1}{15} x\left(t^{2}\right), t \in[0,1], 1<\alpha \leq 2, \\
x(0)=1, x^{\prime}(0)=0 .
\end{array}\right.
$$

where $\alpha=\frac{3}{2}, T=1, \theta(t)=t^{2}$ and $f\left(t, x(t), x\left(t^{2}\right)\right)=\frac{1}{30} x(t)+\frac{1}{15} x\left(t^{2}\right)$. Obviously, $f\left(t, x(t), x\left(t^{2}\right)\right)$ satisfies Lipschitz condition and there exist constants $L_{1}=\frac{1}{30}$, $L_{2}=\frac{1}{15}$ such that

$$
\left|f\left(t, x(t), x\left(t^{2}\right)\right)-f\left(t, y(t), y\left(t^{2}\right)\right)\right| \leq \frac{1}{30}|x(t)-y(t)|+\frac{1}{15}\left|x\left(t^{2}\right)-y\left(t^{2}\right)\right| \text { if } t \in J,
$$

So that condition $\left(H_{1}\right)$ of Theorem 3.1 hold, the problem (12) has a unique solution. Consider the same equation as (12), taking $x_{0}(t)=0, y_{0}(t)=5 t^{-\frac{1}{2}}+6$, and then we have $y_{0}(0)=5$. Moreover,

$$
y_{0}(t)=5 t^{-\frac{1}{2}}+6 \geq 5 t^{-\frac{1}{2}}+\frac{1}{\Gamma\left(\frac{1}{2}\right)} \int_{0}^{t}(t-s)^{-\frac{1}{2}}\left(\frac{1}{30}\left(5 s^{-\frac{1}{2}}+6\right)+\frac{1}{15}\left(5 s^{-\frac{1}{2}}+6\right)^{2}\right) d s,
$$

On the other hand, it is easy to check that $x_{0} \leq y_{0}$ and $\left(H_{2}\right)$ of Theorem 4.1 holds. And let $M=\frac{1}{30}, N=\frac{1}{15}$, we get that

$$
f\left(t, x(t), x\left(t^{2}\right)\right)-f\left(t, y(t), y\left(t^{2}\right)\right) \geq \frac{1}{30}[x(t)-y(t)]+\frac{1}{15}\left[x\left(t^{2}\right)-y\left(t^{2}\right)\right],
$$

where $x_{0} \leq u_{1} \leq v_{1} \leq y_{0}, x_{0}\left(t^{2}\right) \leq u_{2} \leq v_{2} \leq y_{0}\left(t^{2}\right)$. So $\left(H_{3}\right)$ is satisfied. Furthermore, we get that 
Monotone Iterative Technique for Caputo Fractional Differential Equations ...

$$
\frac{(M+N) T^{\alpha}}{\Gamma(1+\alpha)}=\frac{2}{15 \sqrt{\pi}}<1
$$

It is easy to see that (7) holds. Thus, all conditions of Theorem 4.1 are satisfied. Therefore, problem (12) has extremal solutions.

Example 5.2. Consider the following problem:

$$
\left\{\begin{array}{c}
{ }^{C} D_{0+}^{\frac{3}{2}} x(t)=\frac{t^{2}}{60} x(t)+\frac{t^{3}}{30} x\left(\frac{1}{2} t\right), t \in[0,1], 1<\alpha \leq 2 \\
x(0)=1, x^{\prime}(0)=0 .
\end{array}\right.
$$

Where $\alpha=\frac{3}{2}, T=1, \theta(t)=\left(\frac{1}{2} t\right)$ and $f\left(t, x(t), x\left(\frac{1}{2} t\right)\right)=\frac{t^{2}}{60} x(t)+\frac{t^{3}}{30} x\left(\frac{1}{2} t\right)$. Obviously, $f\left(t, x(t), x\left(\frac{1}{2} t\right)\right)$ satisfies Lipschitz condition and there exist constants $L_{1}=\frac{1}{60}$, $L_{2}=\frac{1}{30}$ such that

$$
\left|f\left(t, x(t), x\left(\frac{1}{2} t\right)\right)-f\left(t, y(t), y\left(\frac{1}{2} t\right)\right)\right| \leq \frac{1}{60}|x(t)-y(t)|+\frac{1}{30}\left|x\left(\frac{1}{2} t\right)-y\left(\frac{1}{2} t\right)\right| \text { if } t \in J .
$$

So that condition $\left(H_{1}\right)$ of Theorem 3.1 holds, the problem (13) has a unique solution.

Example 5.3. Consider the following problem:

$$
\left\{\begin{aligned}
{ }^{C} D_{0+}^{\frac{3}{2}} x(t) & =\frac{t^{2}+1}{30} x(t)+\frac{t^{4}+1}{15} x\left(\frac{1}{2} t^{2}\right), t \in[0,1], 1<\alpha \leq 2, \\
x(0) & =1, x^{\prime}(0)=0 .
\end{aligned}\right.
$$

Obviously, $\alpha=\frac{3}{2}, T=1, \theta(t)=\left(\frac{1}{2} t^{2}\right)$ and $f\left(t, x(t), x\left(\frac{1}{2} t^{2}\right)\right)=\frac{t^{2}+1}{30} x(t)+\frac{t^{4}+1}{15} x\left(\frac{1}{2} t^{2}\right)$.

Taking $x_{0}(t)=0, y_{0}(t)=t^{-\frac{1}{2}}+6$, then we have $x_{0}(0)=0, y_{0}(0)=1$. Moreover,

$$
y_{0}(t)=t^{-\frac{1}{2}}+6 \geq t^{-\frac{1}{2}}+\frac{1}{\Gamma\left(\frac{1}{2}\right)} \int_{0}^{t}(t-s)^{-\frac{1}{2}}\left(\frac{\left(s^{2}+1\right)}{30}\left(s^{-\frac{1}{2}}+6\right)+\frac{s^{4}+1}{15}\left(\frac{1}{2}\left(s^{-\frac{1}{2}}+6\right)^{2}\right)\right) d s,
$$

So that condition $\left(\mathrm{H}_{2}\right)$ of Theorem 4.1 holds. On the other hand, it is easy to check that $\left(H_{3}\right)$ of Theorem 4.1 holds, therefore

$$
f\left(t, x(t), x\left(\frac{1}{2} t^{2}\right)\right)-f\left(t, y(t), y\left(\frac{1}{2} t^{2}\right)\right) \geq \frac{1}{30}[x(t)-y(t)]+\frac{1}{15}\left[x\left(\frac{1}{2} t^{2}\right)-y\left(\frac{1}{2} t^{2}\right)\right],
$$

where $x_{0} \leq u_{1} \leq v_{1} \leq y_{0}, x_{0}\left(\frac{1}{2} t^{2}\right) \leq u_{2} \leq v_{2} \leq y_{0}\left(\frac{1}{2} t^{2}\right)$.

We see that $M=\frac{1}{30}, N=\frac{1}{15}$, which satisfied inequality (7). All conditions of Theorem 4.1 are satisfied. So problem (14) has extremal solutions.

Acknowledgements. The first author Dr. D.B.Dhaigude is grateful to the U.G.C. New Delhi for the award of Emeritus Fellowship No.F.6-6/2015-17/EMERITUS-2015-17OBC-7176/(SA-II). 
D. B. Dhaigude and B. H. Rizqan

\section{REFERENCES}

1. J.V.Devi, Generalized monotone method for periodic boundary value problems of Caputo fractional differential equations, Coтmu. Appl. Anal, 12 (4) (2008) 399-406.

2. C.D.Dhaigude, S.P.Bhairat and D.B.Dhaigude, Solution of fractional differential equations involving Hilfer fractional derivative: method of successive approximations, Bull. Marathwada Math. Soc, 18 (2) (2017) 1-12.

3. D.B.Dhaigude, J.A.Nanware and V.R.Nikam, Monotone technique for system of Caputo fractional differential equations with periodic boundary conditions, Dyn. Conti. Dis. Impul. Sys, 19 (2012) 575-584.

4. D. B. Dhaigude and B.H.Rizqan, Existence and uniqueness of solutions for fractional differential equations with advanced arguments, Adv. Math. Models \& Appl, 2 (3) 2017 240-250.

5. T.Jankowski, Fractional differential equations with deviating arguments, Dyn. Syst. Appl, 17 (2008) 677-684.

6. A.Kilbas and S.A.Marzan, Nonlinear differential equations with the Caputo fractional derivative in the space of continuously differentiable functions, Diff. Eqs, 41 (2005) 84-89.

7. A.A.Kilbas, H.M.Srivastava and J.J.Trujillo, Theory and applications of fractional differential equations, North-Holland mathematics studies, 204. Elsevier Science B.V., Amsterdam, 2006.

8. A.A.Kilbas, S.G.Samko and O.I.Marichev, Fractional integral and derivatives, theory and applications, Gordon and Breach, Yverdon, 1993.

9. V.Lakshmikantham, S.Leela and J.V.Devi, Theory of fractional dynamic systems, Cambridge Academic Publishers, Cambridge, 2009.

10. V.Lakshmikantham and A.S.Vatsala, Basic theory of fractional differential equations, Nonlinear Anal, 69 (2008) 2677-2682.

11. V.Lakshmikantham and A.S.Vatsala, Theory of fractional differential inequalities and applications, Commu. Appl. Anal, 11 (3-4) (2007) 395-402.

12. F.Mainardi, Fractional calculus: Some basic problems in continuum and statistical mechanics, Springer-Verlag, Wien, (2012) 291-348.

13. F.A.McRae, Monotone method for periodic boundary value problems of Caputo fractional differential equations, Commu. Appl. Anal, 14 (1) (2010) 73-80.

14. K.S.Miller and B.Ross, An introduction to the fractional calculus and differential equations, John Wiley, New York, 1993.

15. J.A.Nanware and D.B.Dhaigude, Existence and uniqueness of solution of RiemannLiouville fractional differential equations with integral boundary conditions, Int. J. Nonlinear Sci, 14 (2012) 410-415.

16. J.A.Nanware and D.B.Dhaigude, Monotone iterative scheme for system of RiemannLiouville fractional differential equations with integral boundary conditions, Math. Modelling Sci. Comput, 283 (2012) 395-402.

17. J.A.Nanware and D.B.Dhaigude, Existence and uniqueness of solutions of differential equations of fractional order with integral boundary conditions, $J$. Nonlinear Sci. Appl, 7 (2014) 246-254. 
Monotone Iterative Technique for Caputo Fractional Differential Equations ...

18. J.A.Nanware and D.B.Dhaigude, Monotone technique for finite weakly coupled system of Caputo fractional differential equations with periodic boundary conditions, Dyn. Contin. Discrete Impuls. Syst, 22 (1) (2015) 13-23.

19. I. Podlubny, Fractional differential equations, Academic Press, San Diego, 1999.

20. J.Sabatier, O.P.Agrawal and J.A.T.Machado, Advances in fractional calculus: Theoretical developments and spplications in physics and engineering, Springer, Dordrecht, 2007.

21. B.Sambandham and A.S.Vatsala, Basic results for sequential Caputo fractional differential equations, Math, 3 (1) (2015) 76-91.

22. S.G.Samko, A.A.Kilbas and O.I.Marichev, Fractional integrals and derivatives. theory and applications, Gordon and Breach, Yverdon, 1993.

23. A.Yakar, Initial time difference quasilinearization for Caputo fractional differential equations, Adv. Diff. Eqs, 2012 (1) (2012) 2-9. 